\title{
Improve of Health Care Systems for Smart Hospitals Based on UML and XML
}

\author{
Magdy Shayboub Ali Mahmoud ${ }^{1,2}$ \\ ${ }^{1}$ Computer Science Department, Faculty of Computers and Informatics. Suez Canal University, Egypt \\ ${ }^{2}$ Computer Science Department, Faculty of Computers and Information systems \\ Taif University, Kingdom of Saudi Arabia (KSA) \\ magdy01sh $\{$ at $\}$ yahoo.com
}

Abstract-The convergence of information technology systems in health care system building is causing us to look at more effective integration of technologies. Facing increased competition, tighter spaces, staff retention and reduced reimbursement, today's traditional hospitals are looking at strategic ways to use technology to manage their systems called smart hospital. The concept of the smart hospital is a useful system for any hospital; about adding intelligence to the traditional hospital system by covering all resources and locations with patient information. Patient's information is an important component of the patient privacy in any health care system that is based on the overall quality of each patient in the health care system. The main commitment for any health care system is to improve the quality of the patient and privacy of patient's information. Today, there is a need of such computer environment where treatment to patients can be given on the basis of his/her previous medical history at the time of emergency at any time, on any place and anywhere. Pervasive and ubiquitous environment and UML(unified modeling language) can bring the boon in this field. For this it's needed to develop the ubiquitous health care computing environment using the UML with traditional hospital environment. This paper is based on the ubiquitous and pervasive computing environment based on UML and XML(The Extensible Markup Language) technology, in which these problems has been tried to improve traditional hospital system into smart hospital in the near future. The key solution of the smart hospital is online identification of all patients, doctors, nurses, staff, medical equipments, medications, blood bags, surgical tools, blankets, sheets, hospital rooms, etc. In this paper efforts is channeled into improving the knowledge-base ontological description for smart hospital system by using UML and XML technology, Our knowledge is represented in XML format from UML modeling(class diagram). Our smart hospital provides access to its system by using a smart card. Finally, the former try to improve health care delivery through development and management of acute care hospital designed; both physically and operationally, for more efficiency and increased patients safety.

Keywords- UML; Smart Hospital(SH); Ontology; XML; health care system

\section{INTRODUCTION}

With more than 90 percent of hospital administrators involved in constructing a new building or renovating an existing facility to meet the ever-increasing demands for space. Today, traditional hospital executives have to look closer at their work flow processes earlier in the program, in order to capitalize on the latest program technology to optimize clinical, financial and administrative processes. And it involves more than advanced healthcare information systems. It also includes assistance technology such as medical smart card, advanced nurse call, and advanced patient tracking.

There are many organizational units or departments in the traditional hospital, from which, it is necessary for them that there should be good coordination in each other. Even the available health care ontology automation software also does not provide such coordination among them. These software's are limited up to the hospital works but do not provide the interconnectivity with other hospitals and blood banks etc. Thus, these traditional hospitals cannot share information inspite of the good facilities and services.

Many changes and developments in health care environment in the last decades are due to new technologies such as portable devices (Laptop, Mobile) and wireless computing. On the one hand, where the main aim of traditional hospital is to provide better services and facilities to the patient, his/her proper care brings success to the hospital's name. Along with this, traditional hospitals also adds many new facilities and services with existing facilities and services in one place for their patient. Having all facilities and services in the same place, hospital's ability to provide sufficient care to the patient at any place and time.

The major problems with the health care environments are related to the information storage and retrieval of the patient's data and other entities of the health care system. These problems are further categorized below[1]:-

- One problem is when there is information gap among the medical professionals, users/patients and various data source.

- Another problem is that in there is a need to present and organize the information flow among the hospital members and other entities so that information can be accessed at any time and any place.

- Other problems are related to the various types of data used and no common format for holding it in a common way. 


\section{CONCEPT AND DESIGN ONTOLOGY}

The concept of smart hospital has been designed from the ground up to achieve the following goals:

- Safety and clinical quality

- Productivity

- $\quad$ Ease of use for patients, doctors, families and caregivers

- $\quad$ Service excellence

- Optimal use of technologies- medical, information and consumer

There has been much development in the concept of ontology process since the last decade and many good thinkers gave its meaning and its various definitions $[1,6]$. It is a set of primitive concepts that can be use for representing a whole domain or part of it that defines a set of objects, relations between them and subsets of those in the respective domain. It is also a man-made framework that supports the modeling process of a domain in such a way that collection of terms and their semantic interpretation is provided. Our knowledge design in our paper [2] is represented in XML format. In artificial intelligence [13] The term-Ontology is an explicit specification of a conceptualization, where ontology is defined as:

- $\quad$ a vocabulary ; the set of terms used for modeling.

- a structure of the statements in the model.

- the semantic interpretation of these terms.

Ontologies have become ubiquitous [7, 9] in information systems. They constitute the Semantic Web's [4] backbones, facilitate e-commerce, and serve such diverse application fields as bioinformatics and medicine. Many times the meaning of the word 'Ontology' is taken as a branch of philosophy [4] that is the science, of the kinds and structures of objects, properties, events, processes and relations in every area of reality. Sometimes, it is used as a synonym of 'metaphysics' and having broader sense which refers to the study of what might exist and which of the various alternative possible ontologies is in fact true of reality. In simple term, Ontology can be defined as a collection of Classes, Sub-classes that makes the relationship among them and represent the ontology design with knowledge base. Our knowledge base of ontology design is represented in XML format.

\section{SMART HOSPITAL}

The smart hospital accomplishes these goals by taking integrated and current information and communication technologies and combining them with:

- Careful design of the facility to be accessible and efficient,

- Initial engineering and continual redesign of clinical and business processes to operate reliably and safely,

- Constant emphasis on patient and family service and satisfaction, and

- Fervent attention to providing a superior workplace for physicians and staff.

The end result is that smart hospital patients and families experience coordinated, safe, and high quality care in an information-rich, service-minded and easily accessible environment. Physicians affiliated with the smart hospital experience an efficient and clinically focused facility in which to practice state-of-the art evidence-based medicine [24]. Caregivers and technicians at the smart hospital devote time and energy to doing what they do best caring for patients. Figure 1a, show an use-case diagram of a simple smart hospital.

It is a type of hospital that is able to share the domain's knowledge with same or other domain [16] and fulfill the requirement of the ubiquitous and pervasive computing [6] environment. The smart hospital offers a number of advantages:

- It provides a beneficial strategy for the better education and training simulation among the health care professionals.

- It ensures the higher levels of competence, confidence and critical thinking skills.

- It helps to manage the complex and changing health care system.

- It also supports the faculty for developing and evaluating new educational models, modalities, and teaching-learning strategies at no risk to patients.

- It also helps to integrate the better combination of ICT technologies, product and services.

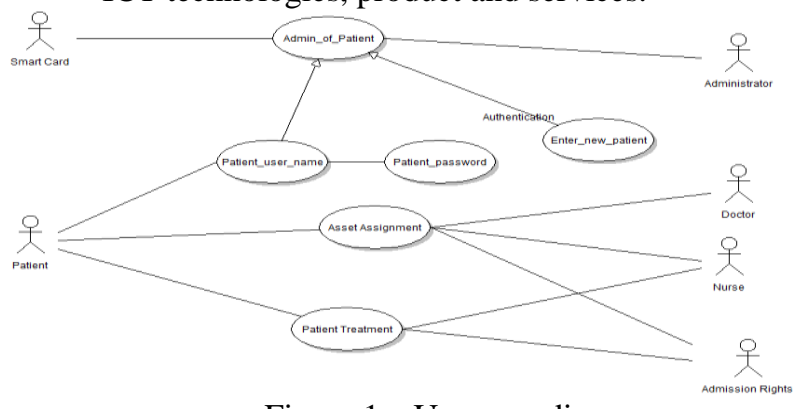

Figure 1a: Use-case diagram

\section{ONTOlOGY DESIGN FOR SMART HOSPITAL (SH)}

The ontology of health care system is a major component where end user interacts with it and the information encompasses a conceptual component i.e. information that plays a role in hospital care outcomes, including errors and difficulties. To deal with the events, Deployment of SH in a particular hospital setting will involve developing the middleware to relate the ontological knowledge base $[1,10]$ with existing information systems and by creating instances of ontological categories that is based on the information in the smart hospital databases [16]. Our knowledge base [1] is represented in XML format from UML modeling. Knowledge representation has been defined as "A set of syntactic and semantic conventions that makes it possible to describe things. The syntax of a representation specifies a set of rules for combining symbols to form expressions in the representation language as shown in figure $1 \mathrm{~b}$. The semantics of a representation specify how expressions so constructed should be interpreted (i.e. how meaning can be derived from a form). In the proposed system, the knowledge representation methodology uses 
XML format. Where, two elements of knowledge, facts and model rules are represented using XML format as shown in figure 2 . The overall knowledge structure is:

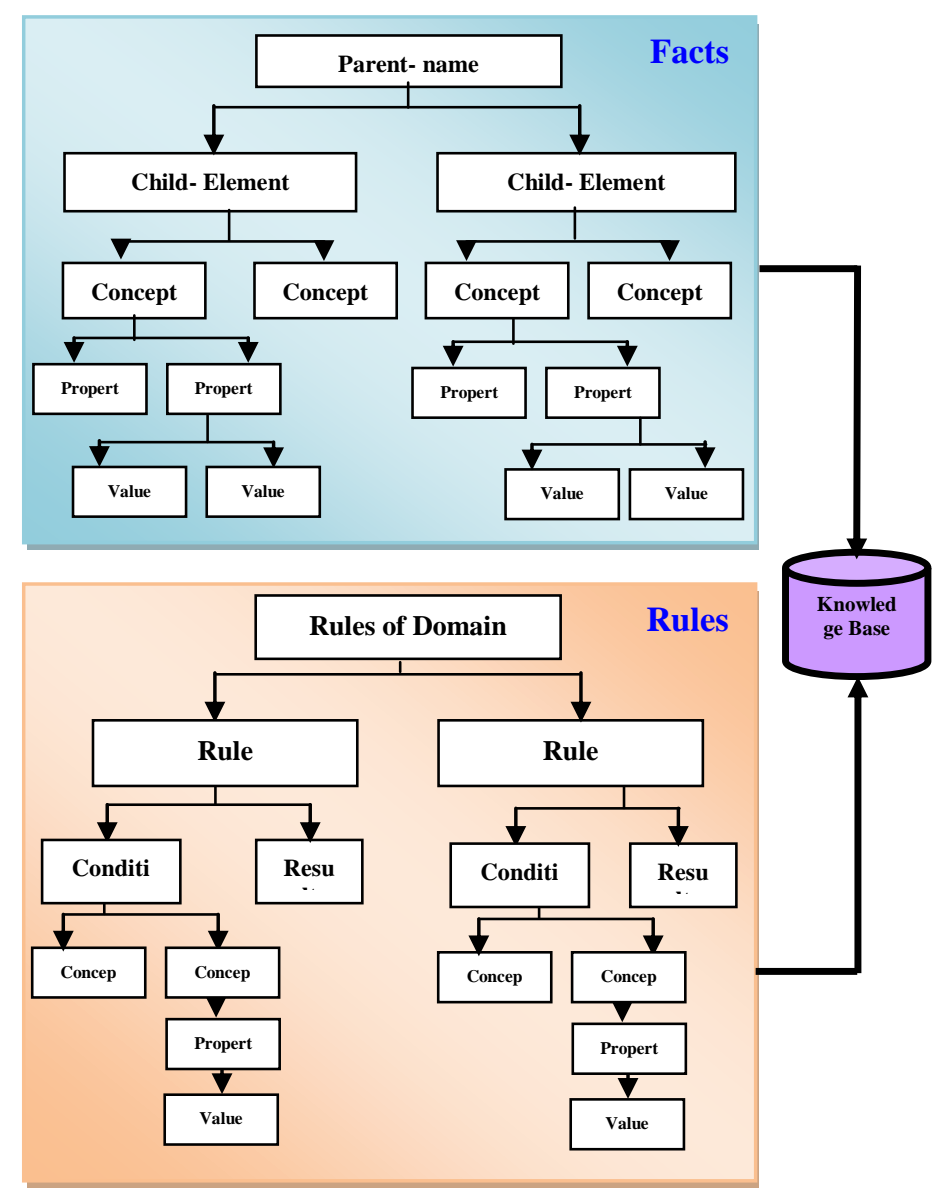

Figure1b: Overall knowledge structure

$<$ Project DefaultTargets="Build" XML

ns="http://schemas.microsoft.com/developer/msbuild/2003"

ToolsVersion="3.5">

$<$ PropertyGroup>

$<$ ProductVersion $>9.0 .21022<$ ProductVersion $>$

$<$ SchemaVersion $>2.0</$ SchemaVersion $>$

$<$ RootNamespace $>$ Untitled $</$ RootNamespace $>$

$<$ AssemblyName>Untitled</AssemblyName>

$<$ ProjectGuid $>\{\}</$ ProjectGuid $>$

$<$ OutputType $>$ Library $</$ OutputType $>$

$</$ PropertyGroup $>$

$<$ PropertyGroup Condition=" '\$(Configuration)|\$(Platform)' ==

'Debug|AnyCPU' ">

$<$ DebugSymbols $>$ true $</$ DebugSymbols $>$

$<$ DebugType $>$ full $</$ DebugType $>$

$<$ Optimize $>$ false $</$ Optimize $>$

$<$ OutputPath $>$ bin $\mid$ Debug $</$ OutputPath $>$

$<$ DefineConstants $>$ DEBUG;TRACE $</$ DefineConstants $>$

$<$ ErrorReport $>$ prompt $</$ ErrorReport $>$

$<$ WarningLevel $>4</$ WarningLevel $>$

$<$ PropertyGroup $>$

$<$ PropertyGroup Condition=" ' $\$($ Configuration $) \mid \$($ Platform $) '==$

'Release|AnyCPU' ">

$<$ DebugType $>$ pdbonly $<$ /DebugType $>$

$<$ Optimize>true</Optimize>

$<$ OutputPath>bin|Releasel</OutputPath>

$<$ DefineConstants $>$ TRACE $</$ DefineConstants $>$

$<$ ErrorReport $>$ prompt $</$ ErrorReport $>$

$<$ WarningLevel $>4</$ WarningLevel $>$
$<$ PropertyGroup $>$

$<$ ItemGroup $>$

$<$ Reference Include="System" />

$\langle$ Reference Include="System.Data" $/>$

$<$ Reference Include="System.XML " / >

$</$ ItemGroup $>$

$<$ ItemGroup >

$<$ Compile Include="PatientSmartCard.cs" $/>$

$<$ Compile Include="Patient.cs" / >

Figure 2:XML Sample of developed facts in our knowledge

\section{SMART Hospital SCHEMA:}

The Smart Hospitals schema uses wireless technology to facilitate communication internally and externally. These schemes can allow a legal user to login to remote server and access its facilities. Its consumer technology improves the flow of information to customers using text messaging pagers, PDAs, tablet PCs, and customer internet access. Electronic medical records, bar coding and integrated vital signs are among the additional technologies that result in such operational efficiencies as:

- $\quad$ Reduced documentation time for nurses, allowing them to spend more time giving patient care.

- Immediate access to diagnostic test results:

- Access to patient medical information by physicians from office, home or elsewhere.

- Improved patient safety through automated checking of medication administration.

- Space savings resulting from digital storage of "films" and other medical records.

- Daily access and feedback to management and physicians of clinical information, financial results and patient satisfaction. 


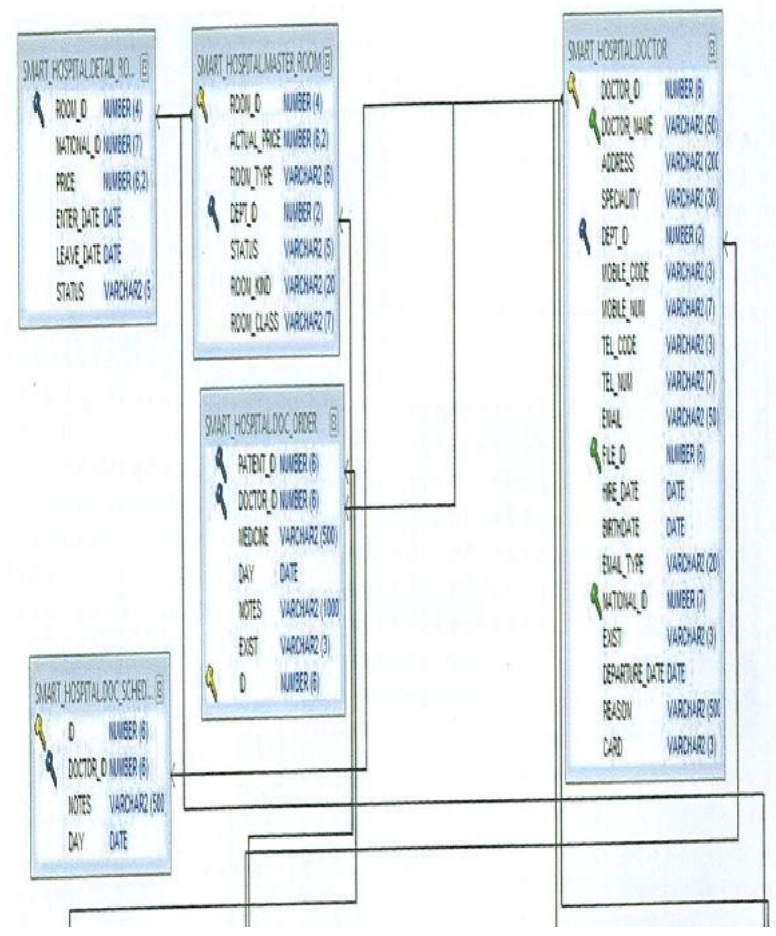

Figure 3a: Smart Hospital Schema 1

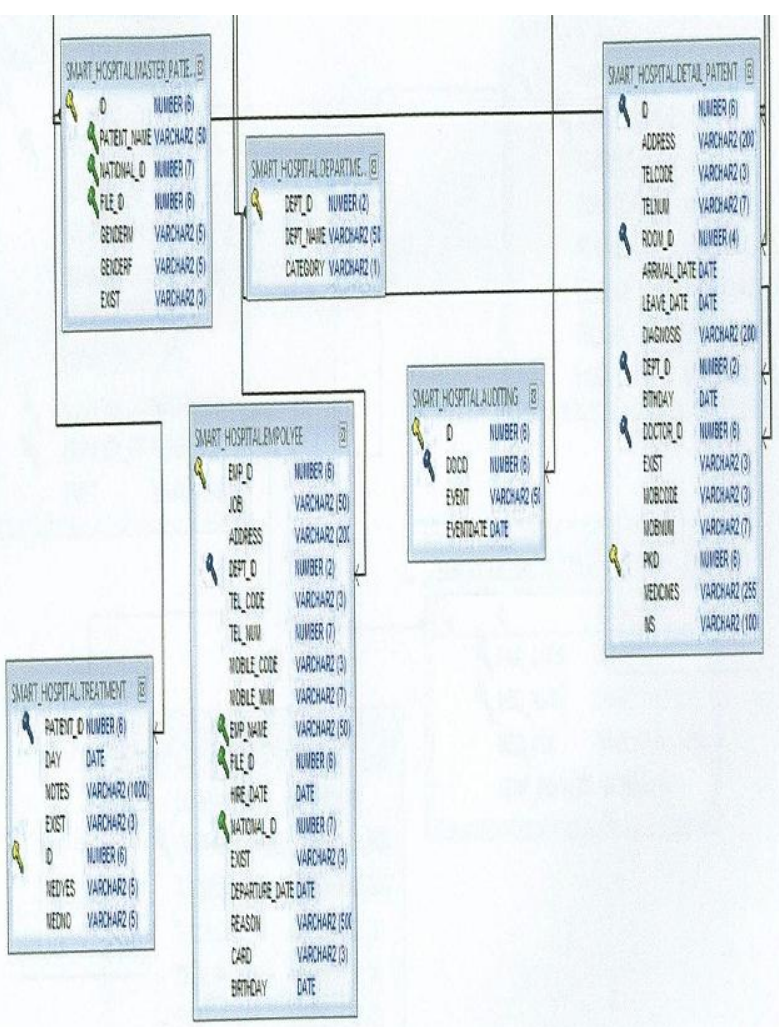

Figure 3b: Smart Hospital Schema 2

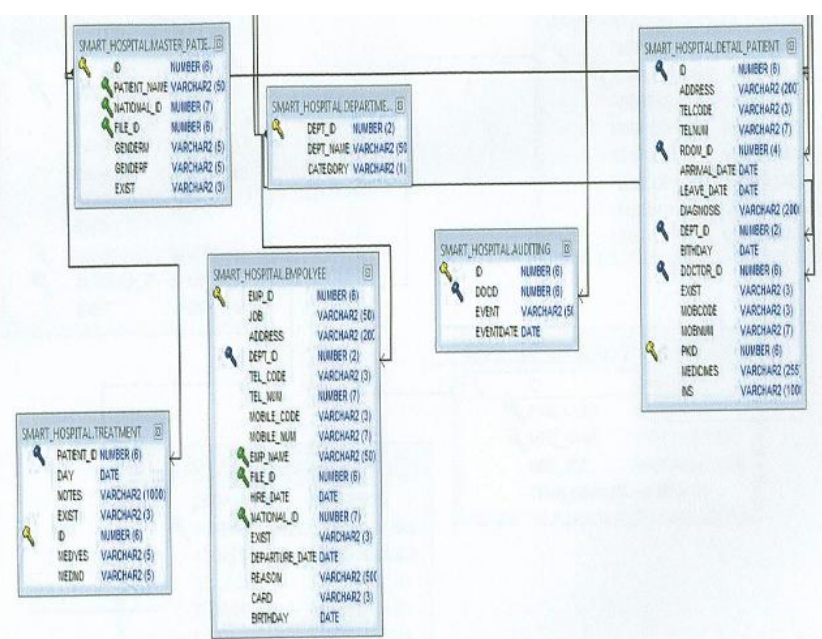

Figure 3c: Smart Hospital Schema 3

In the above figure $3 \mathrm{a}, 3 \mathrm{~b}$ and $3 \mathrm{c}$ show the components of Smart hospital schemas, which are events, actions, person, policies, alerts etc. For example, in the SH different type of objects are taken such as-agents, policies, record, drugs, place and equipment etc.:

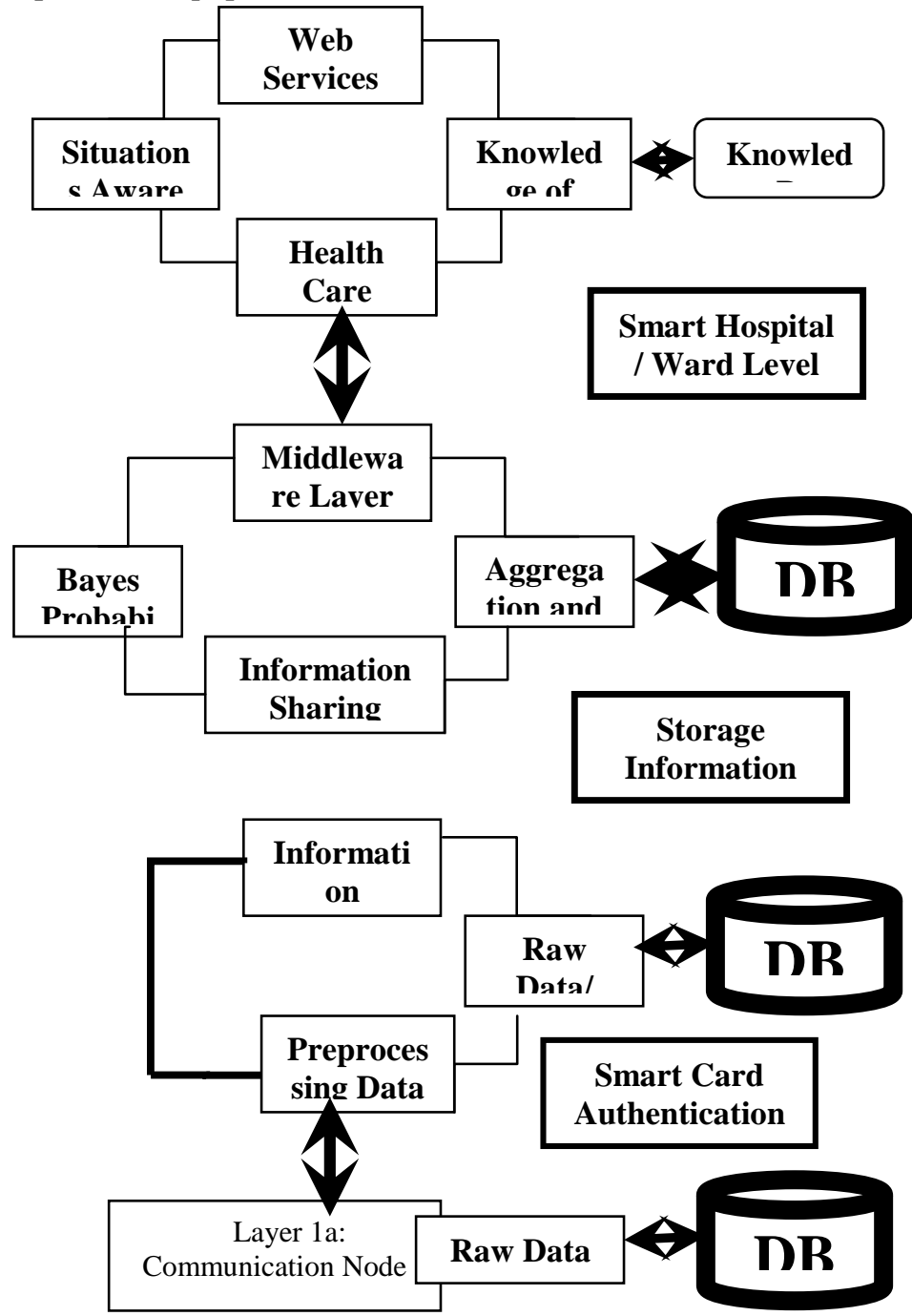

Figure 4: A Middleware Architecture of a patient data collection system for $\mathrm{SH}$ 
Figure 4, illustrates A middleware architecture of a patient data collection system for SH ontology. So, this ontology is able to describe which action and event is performed in what time and what place. This is also useful to alert the different type of domain time to time with different type of alters such as-medical condition alert, medical conflict alert, regulation action and regulatory conflict alert. The major benefits of ontology in health care environment are To find out the common understanding of the structure of information among hospital entities or software agents and share it.

- Domain assumptions can be made explicit.

- To separate domain knowledge from the operational knowledge.

- To analyze domain knowledge

Often ontology of the domain is not a goal in itself. Developing ontology is defining a set of data and their structure for other programs to use. Problem-solving methods, domainindependent applications, and software agents use ontologies and knowledge bases [1] built from ontologies as data. For example, we develop ontology of patient, doctor and nurse and appropriate combinations of patient with doctor and nurse.

\section{METHODLOGY AND APPROACH}

A simple approach used to develop the ontology/knowledge base is the iterative technique that is used to identify the various super classes and sub classes and its properties which is based on the simple knowledge/ontology engineering [16] methodology. This methodology is described as shown in figure 5:

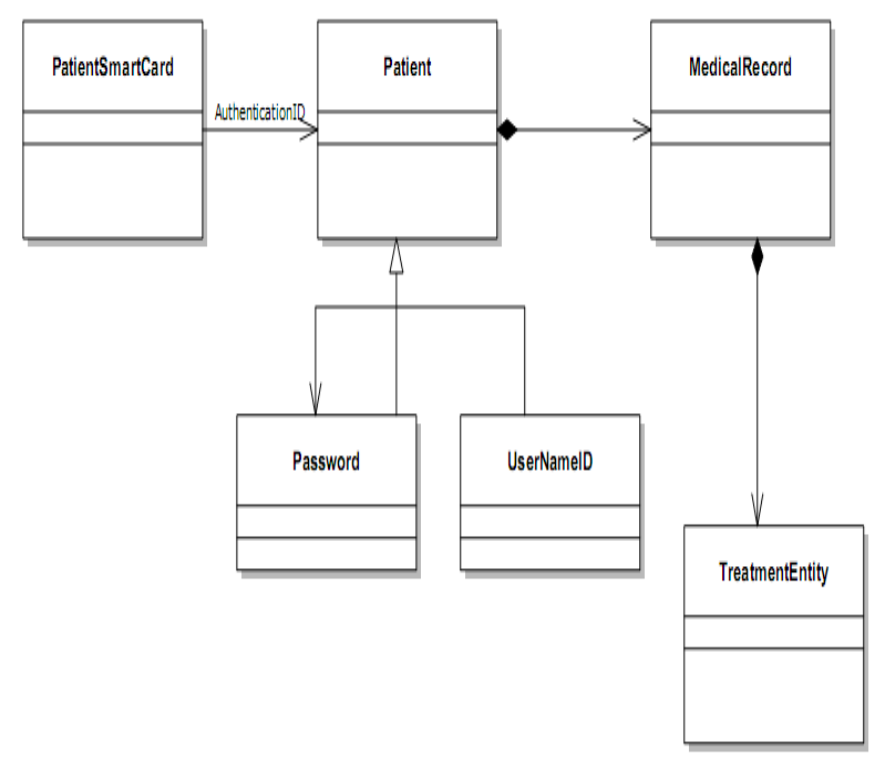

Figure 5: Class Diagram of a Patient Record in SH

\footnotetext{
(A) Knowledge Base Methodology: No specified methods or approaches are still developed for the development of ontology. Our methodology depends on an iterative technique to ontology development. All the steps in this technique are revised and refined in the process of iterative technique to evolve the ontology. The process in iterative design is likely to be continued through the entire development lifecycle of the
}

ontology. Based on the various literature survey, the proposed steps for the processing of developing ontology are:

(i) Finding the domain and scope of the ontology: The first step of the development of ontology is to determine and define its domain and scope. During the determination and definition of it, we must have to consider the following four questions so that we can be able to easily determine it:

- What is the domain that the ontology will cover?

- What are we going to use the ontology?

- What types of questions should the information in the ontology provide answers?

- Who will use and development the ontology?

The answers to these questions may change during the ontologydesign process, but at any given time they help limit the scope of the model. Figure 5, show the class diagram of a Patient Record in smart hospital and the XML format from this figure as shown:

Project DefaultTargets="Build" XML

ns="http://schemas.microsoft.com/developer/msbuild/2003"> $<$ PropertyGroup> $<$ ProductVersion $>8.0 .50727<$ ProductVersion $>$ $<$ SchemaVersion $>2.0</$ SchemaVersion $>$

$<$ RootNamespace $>$ Untitled $</$ RootNamespace $>$

$<$ AssemblyName $>$ Untitled $</$ AssemblyName $>$

$<$ ProjectGuid $>\{\}<$ ProjectGuid $>$

$<$ OutputType $>$ Library $<$ /OutputType $><$ /PropertyGroup $>$

$<$ PropertyGroup Condition=" ' $\$($ Configuration $) \mid \$($ Platform $)$ ' ==

'Debug|AnyCPU' "> < $>$ DebugSymbols $>$ true $<$ /DebugSymbols $>$

$<$ DebugType $>$ full $<$ /DebugType $>$ Optimize $>$ false $</$ Optimize $>$

$<$ OutputPath $>$ bin $\backslash$ Debug $\mid</$ OutputPath $>$

$<$ DefineConstants $>$ DEBUG;TRACE $<$ DefineConstants $>$

$<$ ErrorReport $>$ prompt $<$ /ErrorReport $>$

$<$ WarningLevel $>4<$ /WarningLevel $><$ /PropertyGroup $>$

$<$ PropertyGroup Condition=" ' $\$($ Configuration $) \mid \$($ Platform $)$ ' $==$

'Release|AnyCPU' "> <DebugType >pdbonly</DebugType>

$<$ Optimize $>$ true $</$ Optimize $>$

$<$ OutputPath $>$ bin $\backslash$ Release $</$ OutputPath $>$

$<$ DefineConstants $>$ TRACE $</$ DefineConstants $>$

$<$ ErrorReport $>$ prompt $<$ /ErrorReport $>$

$<$ WarningLevel $>4<$ /WarningLevel $><$ /PropertyGroup $>$

$<$ ItemGroup $>\quad<$ Reference Include $=$ "System" $\mid>\quad<$ Reference Include="System.Data" $><$ Reference Include="System.XML " $\wedge<$ ItemGroup $><$ ItemGroup $><$ Compile

Include="PatientSmartCard.cs" $><$ Compile Include="Patient.cs" > $<$ Compile Include="MedicalRecord.cs" $>>$ Compile Include="Password.cs" $>>$ Compile Include="UserNameID.cs" / $><$ Compile Include="TreatmentEntity.cs" $/></$ ItemGroup $>$ $<$ Import Project="\$(MSBuildBinPath) $\backslash$ Microsoft.CSharp.targets" $/>$ $<$ Project $>$

(ii) Consider reusing existing ontology: The second step to consider is about the existing ontology. The benefit of considering the existing ontology is aout what someone else has done and checking if we can refine and extend existing sources for our particular domain and task. Reusing existing ontology may be a requirement if our system needs to interact with other applications that have already committed to particular ontology or controlled vocabularies. 
(iii) Enumerate important terms in the ontology-preparing vocabulary: The third step is to write down a list of all terms that are used in the system. We need to enumerate all properties that the concepts may have, or whether the concepts are classes or slots.

(iv) Define the classes and the class hierarchy: The forth step is to define the classes and it is hierarchies. There are several possible approaches to develop a class hierarchy. These are: A top-down development process starts with the definition of the most general concepts in the domain and subsequent specialization of the concepts. A middleware development process starts with the definition of the most specific classes, the leaves of the hierarchy, with subsequent grouping of these classes into more general concepts. A mix development process is a combination of the top-down and bottom-up approaches. Here, it is defined as the more salient concepts first and then generalize and specialize them appropriately.

(v) Define the properties of classes: The fifth step is to define the properties of the class. Once we have defined some of the classes, we must describe the internal structure of concepts. For each property in the list, we must determine which class it describes. In general, there are several types of object properties that can become in development of ontology [119-23] .

(B) Define the facets of the rules: The sixth step is to define the new facets of the rules in our knowledge base. These facts can have different from about facets which describe in the value type, allowed values, the number of the values and other features of the values. The last step is to create the individual instances of classes in the hierarchy. Defining an individual instance of a class requires: Choose a class, Create an individual instance of that class, and Filling in the Fact values. The following figures [6.a],[6.b] shows the use of class diagram for patient treatment and Asset Assignment.

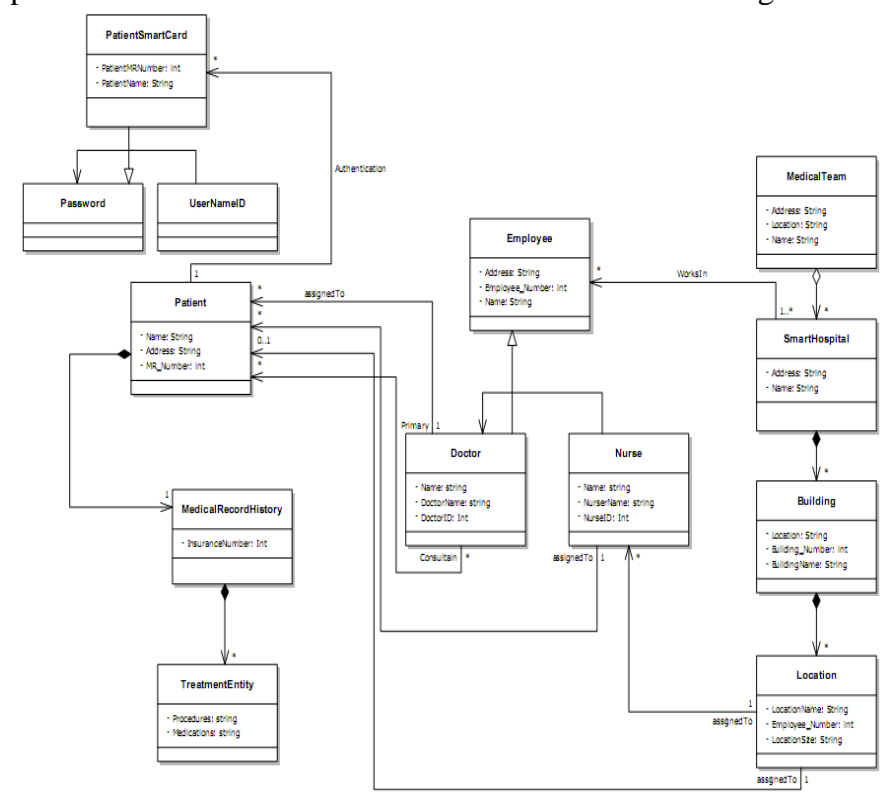

Figure 6(a): Class diagram for patient Treatment.

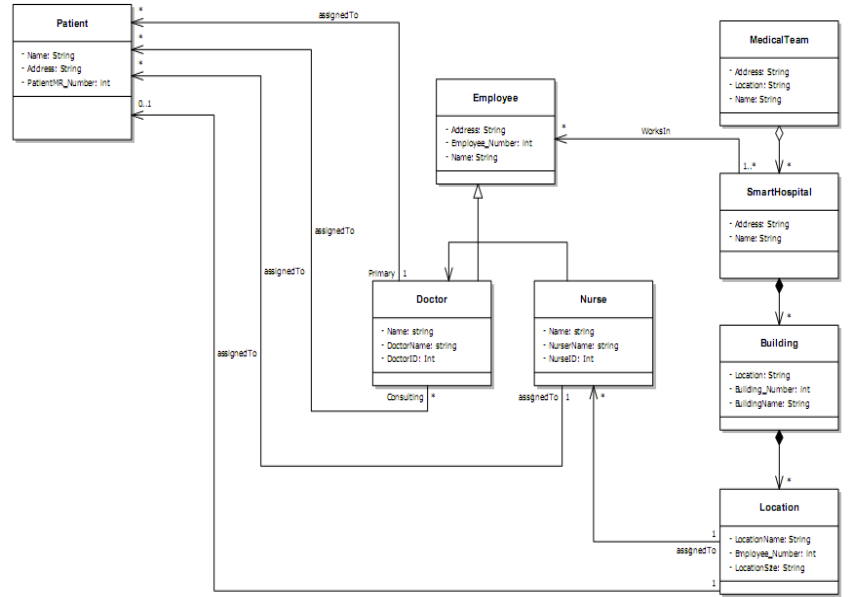

Figure 6(b): Class diagram for Asset Assignment

This UML class diagram provides the graphical representation of visualization, specifying, constructing and documenting the artifacts[19, 22]. The sample of the developed facts in our knowledge is shown in figure 7.

$<$ DiagTestVal $><$ ConceptVal Name="Total Bilirubin $/\rangle\langle$ ValueVal Val="Normal / Increased / $>\langle$ ValueVal Val="Increased $|>$ $</$ ConceptVal> <ConceptVal Name="Conjugated Bilirubin /> $\langle$ ValueVal Val="Increased $/\rangle\langle$ ValueVal Val="Normal $/\rangle$ $</$ ConceptVal $><$ ConceptVal Name="Unconjugated Bilirubin / $>$ $<$ ValueVal Val="Increased $/\rangle\langle$ ValueVal Val="Normal / Increased / $>\langle$ ValueVal Val="Normal $/></$ ConceptVal $></$ DiagTestVal $>$ $>$ DiagConcept $<\quad>$ ResultConcept Name="Prehepatic" NoTrueFinding $=" 1<" \quad>$ TestConcept $\quad \mathrm{Cpt}=$ "Total Bilirubin" Val="Normal / Increased $</ ">$ TestConcept $\mathrm{Cpt}=$ "Conjugated Bilirubin" Val="Increased $</ ">$ TestConcept $\mathrm{Cpt}=$ "Unconjugated Bilirubin" Val="Increased $</ ">$ TestConcept $\mathrm{Cpt}=$ "Urobilinogen" Val="Increased $</$ " $>$ TestConcept $\mathrm{Cpt="Urine} \mathrm{Color"} \mathrm{Val="Normal}$ (urobilinogen $</ "(\quad>$ TestConcept $\quad \mathrm{Cpt}=$ "Stool Color"

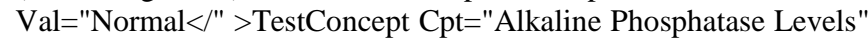
Val="Normal $</ ">$ TestConcept $\mathrm{Cpt}=$ "Alkaline Transferase and Aspartate Transferase Levels" Val="Normal</" >TestConcept $\mathrm{Cpt}=$ "Conjugated Bilirubin in Urine" Val="Not Present $</ "$ 1>ResultConcept< >ResultConcept Name="Hepatic" NoTrueFinding $=4<\quad>$ TestConcept $\mathrm{Cpt}=$ "Total Bilirubin" Val="Increased $</ ">$ TestConcept $\mathrm{Cpt}=$ "Conjugated Bilirubin" Val="Normal $</ ">$ TestConcept $\mathrm{Cpt=}$ "Unconjugated Bilirubin" Val="Normal / Increased $</ ">$ TestConcept Cpt="Urobilinogen" Val="Normal / Increased $</ ">$ TestConcept $\mathrm{Cpt="Urine} \mathrm{Color"}$ Val="Dark (urobilinogen+conjugated bilirubin $</ "$ ( > TestConcept $\mathrm{Cpt}=$ "Stool Color" Val="Normal $</ ">$ TestConcept $\mathrm{Cpt}=$ "Alkaline Phosphatase Levels" Val="Increased $</$ " >TestConcept $\mathrm{Cpt}=$ "Alkaline Transferase and Aspartate Transferase Levels" Val="Increased $</ ">$ TestConcept $\mathrm{Cpt}=$ "Conjugated Bilirubin in Urine" Val="Present $</$ " $/>$ ResultConcept $<$

Figure 7: Sample of developed Rules in our knowledge.

In our domain knowledge [1] the facts is represented as shown in figure 7 where the concept "Total Bilirubin" is a one of the diagnostic test for "Jaundice" and the concept has possible values are " Normal / Increased " and " Increased ". The property of each concept here is default as "Value". The 
knowledge can be formulated as shown in the following simple statements: IF the 'traffic light' is green THEN the action is go, as for example: IF the 'traffic light' is red THEN the action is stop. These statements represented in the IF-THEN form are called production rules or just rules. The term 'rule' in artificial intelligence, which is the most commonly type of knowledge representation, can be defined as IF-THEN structure that relates given information or facts in the IF part to some action in the THEN part. A rule provides some description of how to solve a problem. Rules are relatively easy to create and understand. Any rule consists of two parts: the IF part, called the antecedent (premise or condition) and the THEN part called the consequent (conclusion or action). The basic syntax of a rule is: IF < antecedent> THEN <consequent>. The rules in XML format have a different structure with the previous meaning but in different format. Sample of rule built in the proposed HCS system is shown below; it can be interpreted as following:

$<$ DiagConcept>; represents the root in the domain of the Jaundice.

The node of "ResultConcept" represents a rule consequent and has attribute "Name" its value takes the consequent as" Prehepatic ".

The child nodes "TestConcept" represent the decision rule for each part in Jaundice diagnosis that has two attributes are " Cpt ", and "Val". For example the antecedent of rule is " Total Bilirubin = Normal $/$ Increased ".

The attribute "NoTrueFinding" represents the number of rule antecedent selected.

\section{IMPLEMENTATION MODEL OF SMART HOSPITAL}

It is a useful system for any hospital; especially, Smart hospital concerning with all aspects and issues related to a hospital included patients, doctors, employees, treatment and departments. By using internet, doctors can access the system from any place around the world as shown in an assembled pictures below. Figure 8, illustrates the collection steps for Implementation of our smart Hospital model by using medical smart card to development the ontology system in modern hospital (smart hospital). Our smart hospital provides access to its system by using a smart card through three levels to maintain:

-Write.

$\cdot$.Modify.

$\cdot$.Full control
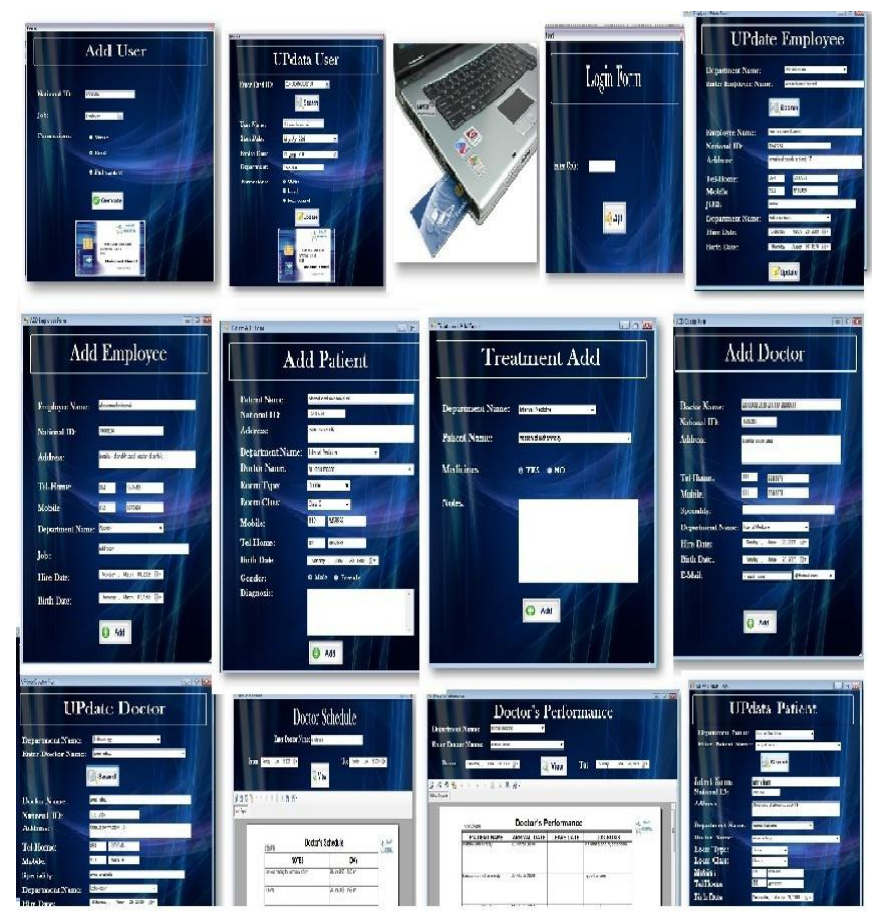

Figure 8: Implementation of our smart Hospital model.

\section{CONCLUSION}

Today's hospitals are looking at strategic ways to use technology to manage their systems called smart hospital. The concept of the smart hospital is about adding intelligence(smart) to the traditional hospital system by covering all resources and locations with patient information's. Patient's information is an important component of the patient privacy in any health care system that is based on the overall quality of each patient in the health care system. The main commitment for any health care system is to improve the quality of the patient and privacy of patient's information. For this, it is needed to develop the ubiquitous health care computing environment using the XML technology with traditional hospital environment.

This paper is based on the UML and XML technology to design and development of the ontology system, can be solved these problems has been tried to improve traditional hospital system into smart hospital. The key solution of the smart hospital is online identification of all patients, doctors, nurses, staff, Medical equipments, medications, blood bags, surgical tools, blankets, sheets, hospital rooms, etc. In this paper, Efforts are to improve the knowledge-base ontological description for smart hospital system by using UML and XML technology, Our knowledge is represented in XML format from UML modeling(class diagram). Finally, we implementing our model in smart hospital by using a medical smart card to improve the performance of health care system. 


\section{REFERENCES}

[1] Sanjay Anand and Akshat Verma " Development of Ontology for Smart Hospital and Implementation using UML and RDF, IJCSI International Journal of Computer Science Issues, Vol. 7, Issue 5, p.p. 206-212, September 2010.

[2] Khaled M Fouad, Magdy Shayboub and Yasser N. "Agent of Healthcare system based on XML Technology "International Journal of Computer Information Systems, Vol. 2, No. 1, szlssa pp.35-4, 2011

[3] Bardram JE. and Christensen HB. "Pervasive Computing Support for Hospitals: An overview of the Activity-Based Computing Project," IEEE Pervasive Computing, Vol. 16, No. 1, pp. 44-51, 2007.

[4] Berners-Lee, Tim, James H, \& Ora L. "The Semantic Web," Scientific American, May 2001.

[5] Horrocks I; Patel-Schneider PF.; and van Harmelen F. Reviewing the design of DAML+OIL: An ontology language for the semantic web. In Proc. of the 18th Nat. Conf. on Artificial Intelligence (AAAI 2002).

[6] Grainger M; Lee J "Ontology - Applications and Design. Communications of the ACM", Vol. 45, No. 2, February 2002.

[7] Weiser M "Ubiquitous Computing". IEEE Computer, 26(10):71-72, 1993.

[8] Erdmann M and Studer R. "Ontology as conceptual models for XML documents". In Proceedings of the 12th Workshop on Knowledge Acquisition, Modeling and Management (KAW'99). Knowledge Science Institute, University of Calgary, 1999. http://sern.ucalgary.ca/KSI/KAW/KAW99/papers/Erdm ann1/erdmann.pdf.

[9] Dean M; Schreiber G; Bechhofer S; van Harmelen F; Hendler J; Horrocks I; McGuinness DL; Patel-Schneider PF; and Stein L A (2004). OWL web ontology language reference. W3C Recommendation. http://www.w3.org/TR/owl-ref/

[10] Alani H, Kim S, Millard DE, Weal MJ, Hall W, Lewis PH and Shadbolt NR. "Automatic Ontology-Based Knowledge Extraction fromWeb Documents",IEEE Intelligent Systems, Vol. 10,No1, pp.14-2, 2003..

[11] Guided Tour of Ontology; John F. Sowa, http://www.jfsowa.com/ontology/guided.htm

[12] Gandon F. Ontology Engineering: a survey and a return on experience, Research Report of INRIA $n^{\circ} 4396$, France, March 2002.

[13] AIWatch-TheNewsletter of Artificial Intelligence.Vol. 46.AI Intelligence, Oxford,UK.

[14] Uschold $M$ and Gruninger M. Ontologies: Principles, methods and applications. Knowledge Engineering Review,
Vol. 11:2, 93-136, 1996. Also available as AIAI-TR-191 from AIAI, the University of Edinburgh.

[15] Bruno B. "Modélisation linguistique et modélisation logique des ontologies: l'apport de l'ontologie formelle." In Proceedings of IC 2001, pp 349-368 Plate-forme AFIA, Grenoble 25-28 juin 2001.

[16] Press Release -The Smart Hospital at The University of Texas at Arlington School of Nursing becomes a Laerdal Center of Excellence in Simulation. Available athttp://www.uta.edu/nursing/simulation/pr-laerdal.pdf

[17] Victor Foo Siang Fook et al., An ontology-based Context Model in Monitoring and Handling Agitation Behaviour for Persons with Dementia, Ubicare Workshop for IEEE International Conference on Pervasive and Communications.2006.

[18] Bardram J. Applications of context-aware computing in hospital work - examples and design principles, Proceedings of the ACM Symposium on Applied Computing. 2004.

[19] Favela et al., "Activity Recognition for Context-Aware Hospital Applications: Issues and Opportunities for the Deployment of Pervasive Networks," Mobile Networks and Applications, vol. 12, nos. 2-3, pp. 155-171, 2007.

[20] Rodriguez et al.,. "Agent-Based Ambient Intelligence for Healthcare,” AI Comm., vol. 18, no. 3, pp. 201-216, 2005.

[21] Moran et al., "Mobility in Hospital Work: Towards a Pervasive Computing Hospital Environment," Int'l J. Electronic Healthcare, vol. 3, no. 1, pp. 72-89, 2006.

[22] V Stanford "Beam Me Up, Doctor McCoy," IEEE Pervasive Computing, vol. 2, no. 3, pp. 13-18, 2003.

[23] D Sánchez, M Tentori and J Favela "Hidden Markov Models for Activity Recognition in Ambient Intelligence Environments," Proc. 8th Mexican Int'l Conf. Current Trends in Computer Science (ENC 07), IEEE CS Press, pp. 33-40, 2007.

[24] Manhattan Research inc. , "Physicians in 2012: The Outlook for On Demand, Mobile, and Social Digital Media". A Physician Research Module Report, 2009, New York, USA

\section{About Author}

Magdy Shayboub Was born in El- Menoufia, Egypt, in 1966. He received the B.Sc. degree in Electronic Engineering in 1989, and M.Sc. degree for his work in Computer Science and Engineering in 1998, all from the Faculty of Electronic Engineering, Menoufia University, Egypt. In 2005, he received his $\mathrm{Ph}$. D. in Computer Science from Faculty of Computers and Informatics from Helwan University, Egypt. He is working as an assistant professor of computer science at the Faculty of Computers and Informatics, Suez Canal University, Egypt from 2006 until now. His research interests are in Artificial Intelligent (AI) Applications and Intelligent Agents (IA), Information Retrieval, Computer Networks Security and Semantic Web. Now is working a head of Medical Records Department and computer science in the Faculty of applied Medical Science in Taif University, Kingdom of Saudi Arabia. 